\title{
Cochlear dysfunction and microvascular complications in patients with type 1 diabetes mellitus
}

João Soares Felício*, Lilian de Souza d'Albuquerque Silva, Carlliane Lima e Lins Pinto Martins, João Felício Abrahão Neto, Manuela Nascimento de Lemos, Fabrício de Souza Resende, Wanderson Maia da Silva, Angélica Leite de Alcântara, Maria Clara Neres lunes de Oliveira, Norberto Jorge Kzan de Souza Neto, Isabela Imbelloni Farias de Franco, Nathalie Abdallah Zahalan, Luísa Correa Janaú, Ana Carolina Contente Braga de Souza, Flavia Marques Santos, Natércia Neves Marques de Queiroz, Neyla Arroyo Lara Mourão, Márcia Costa dos Santos, Karem Miléo Felício and Franciane Trindade Cunha de Melo

\begin{abstract}
Sensorineural hearing impairment has been associated with DM, and it is probably linked to the same pathophysiological mechanisms as well-established in microvascular diabetes complications. The study of otoacoustic emissions (OAEs) is useful to identify subclinical cochlear dysfunction. Therefore, the aim of this study was to evaluate the association between abnormal OAEs responses, diabetic kidney disease (DKD) and diabetic cardiac autonomic neuropathy (CAN). We performed a cross-sectional study with 37 type 1 DM patients without auditory symptoms, submitted to the study of Distortion Product Otoacoustic Emissions (DPOAEs) and screened for DKD and CAN. The otoacoustic emissions responses were considered abnormal in 27/37 (73\%) patients. A correlation was found between abnormal OAEs responses and presence of DKD $(r=0.36, p<0.05)$, and 14/16 (88\%) patients with a lower amplitude of OAEs in $8 \mathrm{kHz}$ frequency band presented DKD. Abnormal OAEs responses in the $6 \mathrm{kHz}$ frequency band were correlated with the presence $(r=0.41, p=0.01)$ and severity of CAN $(r=0.44, p<0.001)$. Additionally, 7/9 (78\%) patients with abnormal OAE responses in this frequency also presented abnormal CAN scores. Our results suggest that abnormal otoacoustic emissions responses in high frequency bands are associated with diabetes microvascular complications and could be a risk marker for DKD and CAN, presenting low sensitivity and high specificity. Therefore, assuming that hearing impairment is a pre-clinical stage of hearing loss, performing distortion product otoacoustic emissions in T1DM patients with microvascular complications could be useful to identify those who would be benefit with regular audiologic follow up and tighter diabetes control.
\end{abstract}

Keywords: Type 1 diabetes mellitus, Cochlear dysfunction, Sensorineural hearing loss, Otoacoustic emissions, Diabetic kidney disease, Cardiac autonomic neuropathy

\footnotetext{
*Correspondence: felicio.bel@terra.com.br

Endocrinology Division - Programa de Pós-Graduação em Oncologia e Ciências Médicas, University Hospital João de Barros Barreto, Federal University of Pará, Mundurucus Street, 4487, Guamá, Belém, PA Postal Code: 66073-000, Brazil
} 


\section{Introduction}

An association between diabetes mellitus (DM) and sensorineural hearing impairment has been widely discussed, but despite a large number of studies performed, it remains controversial [1-5]. Hearing loss is very prevalent among the elderly population, however it is almost twice as common in diabetic adults [6]. Several studies also demonstrated a high prevalence of hearing impairment in diabetic patients, suggesting that DM may be an independent risk factor for hearing loss, justifying screening for this condition in these patients [6-9].

It is well known that DM is associated to many microvascular complications. Human cochlea has an extensive microvasculature and it is considered vulnerable to the effects of microangiopathy, one of the hyperglycemia consequences. Hearing loss in DM may be a result of microangiopathy and several studies postulate this [10-12]. The study of otoacoustic emissions (OAEs) is a simple, objective, highly reproducible, frequency-specific and noninvasive method for the evaluation of the cochlear micromechanics $[13,14]$, and is very useful to identify subclinical sensorineural hearing impairment $[15,16]$, which is more frequently found by distortion product of otoacoustic emissions (DPOAE) in diabetic patients [17]. The aim of the present study is to evaluate the association between abnormal otoacoustic emissions responses, DKD and CAN in patients with type 1 diabetes mellitus (T1DM) without auditory symptoms.

\section{Methods}

\section{Study design and patients}

We performed a cross-sectional study in 37 patients with T1DM. Age under 8 years old, patients with any acute or chronic otological pathology, personal or family history of hearing loss, use of ototoxic drugs, excessive exposure to noise, presence of infection or other urinary tract diseases and any known cardiac diseases were exclusion criterias. This study was developed according to Declaration of Helsinki and the Nuremberg Code and was approved by the University Hospital João de Barros Barreto ethics committee, protocol number 236/08. Signed consent was obtained from all patients.

\section{Clinical and laboratory data}

Medical records analysis and physical examination were performed. Information on demographics, physical measures, pre-existing clinical conditions, duration of T1DM (in years), current medications and body mass were analyzed. Laboratory tests were also performed and included measurements of fasting plasma glucose (FPG), total cholesterol, LDL cholesterol, HDL cholesterol, triglycerides, thyroid-stimulating hormone (TSH), free thyroxine (FT4), serum creatinine and glycated hemoglobin (HbA1c) by high-performance liquid chromatography method (HPLC). GFR was calculated using and modification of diet in renal disease (MDRD) equations.

In addition, we evaluated the presence of DKD, obtaining at least three 24-h urine samples for measuring albuminuria (by immunoturbidimetry). After these initial procedures, all patients underwent routine otorhinolaryngological evaluation with otoscopy, followed by acoustic reflexes assessment, vocal and pure tone audiometry, tympanometry and Distortion Product Otoacoustic Emissions (DPOAEs). We have excluded from our analysis any patient who had current or medical history of otological problems. Additionally, the subjects were screened for CAN.

\section{Otoacoustic emissions}

OAEs are sounds caused by the motion of the cochlea's sensory hair cells as they energetically respond to auditory stimulation, they are recorded by a probe containing a microphone transducer inserted into the ear canal. Separate responses from different parts of the cochlea are obtained by splitting the response into frequency bands. Therefore, otoacoustic emissions are frequency-specific responses and tend to emerge only in frequency bands in which hearing is near normal, providing a useful pointer to normally and abnormally functioning parts of cochlea [18].

The method of OAEs recording used in this study was the Distortion Product Otoacoustic Emissions (DPOAEs), which uses tonal stimulation, with a wider frequency range observation and wide-spread clinical use $[18,19]$.

The frequencies analyzed in this study were $0.5 \mathrm{k}, 1 \mathrm{k}$, $2 \mathrm{k}, 4 \mathrm{k}, 6 \mathrm{k}$ and $8 \mathrm{k} \mathrm{Hz}$. The test was considered abnormal when the signal-to-noise ratio (SNR) was below $6 \mathrm{~dB}$ in at least one of the frequencies assessed [20].

\section{Diabetic kidney disease}

DKD was diagnosed after we evaluated the presence of micro or macroalbuminuria in at least two of the three 24-h urine samples, according to ADA recommendation [21]. Microalbuminuria was defined as a urinary albumin excretion rate (UAER) in the range of $30-300 \mathrm{mg} / 24 \mathrm{~h}$, and macroalbuminuria, as UAER equal to or greater than $300 \mathrm{mg} / 24 \mathrm{~h}$. However, nondiabetic renal disease has been significantly found in diabetic patients, either alone or combined with DKD, in a recent study [22]. Therefore, our data should be analyzed carefully. Additionally, we performed urinalysis, urine culture and an ultrasound scan of the urinary tract to evaluate the presence of infection or other urinary tract conditions that might interfere with the study results. 


\section{Diabetic cardiac autonomic neuropathy}

Tests were conducted in both groups as proposed by Ewing et al. [23] and Valensi et al. [24]. They were based on the response of the heart to the Valsalva manoeuvre (Valsalva ratio), variation in heart rate ( $R R$ interval) during deep breathing (deep breathing test) and response of BP to the change recumbent from lying position to standing position (lying-to-standing test). The first two tests reflect parasympathetic integrity, and the last reflects sympathetic integrity.

\section{Deep breathing test}

Increases in heart rate ( $R R$ interval) while breathing were measured during the deep breathing test. The heart rate usually depends on parasympathetic nervous system integrity. Patients with diabetic CAN have a considerable reduction or even complete absence of increases in heart rate. Patients stayed in the supine position, were quiet and took six deep breaths in $1 \mathrm{~min}$ ( $5 \mathrm{~s}$ for inspiration and $5 \mathrm{~s}$ for expiration) while an electrocardiogram was recorded, using a marker to indicate the end of each inspiration and expiration. Increases in heart rate during the breathing were recorded (normal heart rate increase was defined as at least 15 beats/ $\mathrm{min}$, 'borderline' as 11 beats/min to 14 beats/min, and abnormal as 10 beats/min or less) [25].

Valsalva ratio (cardiac response to valsalva manoeuvre): During the effort period, BP falls and the heart rate should rise. After resting, the BP rises, overtaking its normal rest value, and the heart rate decreases. The test consisted of forced exhalation and maintaining a pressure of $40 \mathrm{mmHg}$ for $15 \mathrm{~s}$ while an electrocardiogram was recorded. It was performed three times in the space of $1 \mathrm{~min}$, with the patient resting between each test. The results were expressed as a Valsalva ratio, which is the ratio between the highest $R R$ interval after the manoeuvre (reflecting bradycardia following the relaxation) and the lowest $R R$ interval during the manoeuvre (which reflects tachycardia during exercise). The test was performed three times and the mean value for the ratio was used. Valsalva ratios of 1.21 or greater were defined as normal, 1.11-1.20 as 'borderline' and 1.10 or less as abnormal.

\section{Lying-to-standing test}

This test was performed analysing variation in blood pressure from lying to standing positions, as previously described. Decreases $>20 \mathrm{mmHg}$ in systolic blood pressure were considered abnormal [23, 24, 26]. Reproducibility of these methods has been demonstrated in diabetic patients. The coefficients of variation are $9.2 \%$, $12.6 \%$ and $6.4 \%$ for the Valsalva manoeuvre, deep breathing test and lying-to standing test, respectively [27].
For statistical analysis, the severity of CAN was measured by a score assigned to each patient according to the results obtained in the tests, as follows: 0 -all tests resulted in normal values; 1 -only one test resulted in abnormal values; 2 -two tests resulted in abnormal values; and 3-all tests resulted in abnormal values. Individuals with test results classified as borderline received a score of 0.5 for the corresponding test. One abnormal test or two borderline results in different tests were considered as CAN for statistical analysis.

\section{Statistical analysis}

Categorical variables were described as frequency (percentage). Numeric variables with normal distribution were described as mean (SD) and non-normally distributed as median (minimum-maximum). Chi square and Fisher's exact test were used to compare categorical variables. The Student's t-test and Mann-Whitney test were used to compare subgroups with and without normal distributions, respectively. To establish correlations between variables, Pearson and Spearman tests were used. The analysis of variance compared more than two subgroups with normal distribution, and the Kruskal-Wallis test was used to compare more than two subgroups without normal distribution. Sensitivity, specificity and accuracy to the cut-off points previously established were estimated based on screening test. The best cut-off point was defined based on the Youden Index (J) and, additionally, a ROC (Receiver Operating Characteristic) Curve was constructed. The cut-off with maximum sensitivity and specificity in the ROC curve was defined as the minimum value in the equation $\left[(1-\text { sensitivity })^{2}+(1-\right.$ specificity $\left.)^{2}\right]$ and the accuracy was estimated based on the area under the ROC curve. Predictive values and likelihood ratios were also calculated from the values of sensitivity and specificity. The ROC curve was built with $8 \mathrm{kHz}$ frequency bands in $\mathrm{dB}$ and the presence or not of DKD, based on ADA criteria. All tests were performed using the SPSS ${ }^{\circledR}$ statistics software. Further, the results were considered significant if $\mathrm{p}$-value $\leq 0.05$.

Interferences are represented by hypothesis tests with a bilaterally significance level of 0.05 . All information was stored and processed with the software SigmaStat (Jandel Scientific) version 3.5 and SPSS (Statistical Package for Social Sciences) 22.0 (IBM).

\section{Patient and public involvement}

Our research question was developed based on previous studies that have already found auditory damage as a DM complication, related to microvascular physiopathology. Furthermore, patients were included in this study as samples, and were not involved in recruitment and conduct of this study. Informed consent was 
obtained from all of them and they had access to the results of this research. T1DM patients who had abnormal OAE responses are still being followed in our center with special attention in diabetic kidney disease and cardiac autonomic neuropathy.

\section{Results}

The clinical and laboratory data of the patients are described in Table 1 . Three patients presented hypertension (7.7\%), 5 had dyslipidemia (12.8\%) and 3 presented hypothyroidism (7.7\%). The individuals with hypothyroidism were using a stable dose of levothyroxine and had normal thyroid function. All patients were in insulin therapy and 7 (17.9\%) also used metformin. Only one patient reported smoking.

\section{Otoacoustic emissions and audiometry}

Only 10/37 (27\%) patients had normal Distortion Product Otoacoustic Emissions in all frequency bands. The OAEs responses were considered abnormal in 27/37 (73\%) patients.

The frequency-specific responses are presented in Fig. 1. Fourteen patients had abnormalities in two or more frequency bands.

Abnormal audiometry was observed in 6/37 (16\%) patients and it was correlated with the CAN score $(\mathrm{r}=0.42, \mathrm{p}<0.05)$. There was no correlation between auditory impairment by audiometry and DKD.

\section{Otoacoustic emissions and DKD}

DKD was observed in 21/37 (57\%) patients and just two of them presented macroalbuminuria. GFR and UAER are described in Table 2.

Abnormal OAEs responses were correlated with the presence of DKD $(r=0.36, p<0.05)$ and UAER

\section{Table 1 Clinical and laboratorial data of patients with T1DM}

\begin{tabular}{ll}
\hline Variables & \\
\hline Age (years) & $23 \pm 8$ \\
Sex (F/M) & $20 / 17(54 / 46 \%)$ \\
Duration of diabetes (years) & 10.2 (2 to 24) \\
HbA1c (\%) & $9.4 \pm 2.5$ \\
SBP $(\mathrm{mmHg})$ & $109 \pm 14$ \\
DBP $(\mathrm{mmHg})$ & $71 \pm 10$ \\
BMl ( $\left.\mathrm{kg} / \mathrm{m}^{2}\right)$ & $23 \pm 3$ \\
Total cholesterol (mg/dl) & $185 \pm 63$ \\
HDL cholesterol (mg/dl) & $49 \pm 13$ \\
LDL cholesterol (mg/dl) & $112 \pm 51$ \\
Triglycerides (mg/dl) & $115 \pm 78$
\end{tabular}

HbA1c, glycated hemoglobin; SBP, systolic blood pressure; DBP, diastolic blood pressure; $\mathrm{BMI}$, body mass index

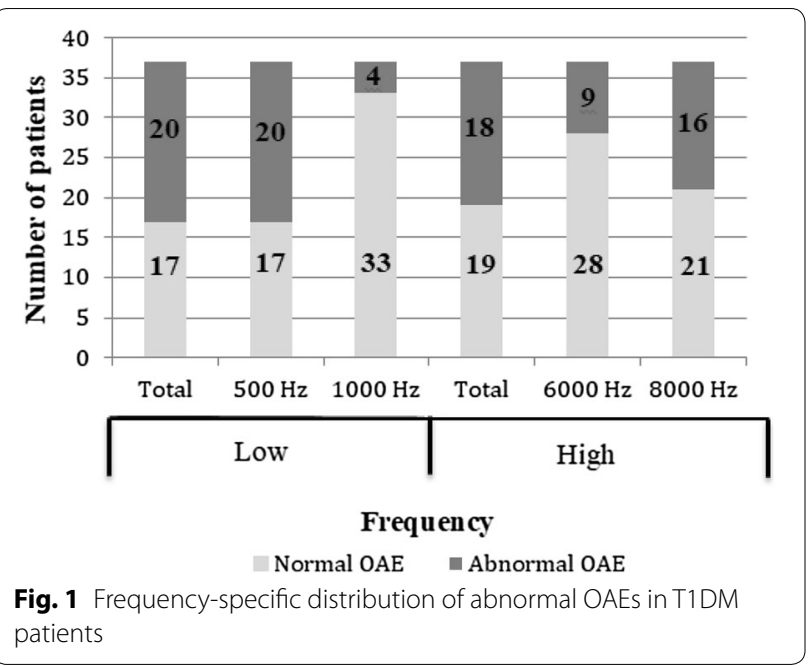

$(\mathrm{r}=0.34, \mathrm{p}<0.05)$. We have found UAER of $96 \pm 137$ and $157 \pm 404 \mathrm{mg} / 24 \mathrm{~h}$ in patients with normal and abnormal otoacoustic emissions responses, respectively; however, it was not statistically significant.

Additionally, we have observed a correlation between the SNR of OAEs in the $8 \mathrm{k} \mathrm{Hz}$ frequency band and UAER $(\mathrm{r}=-0.37, \mathrm{p}<0.05)$ (Fig. 2). Moreover, among the 16 patients with abnormal otoacoustic emissions responses in this frequency band, 14 (88\%) presented DKD.

As screening test to DKD, in $8 \mathrm{k} \mathrm{Hz}$ frequency band, the study of Distortion Product Otoacoustic Emissions showed $30 \%$ of sensitivity and $85 \%$ of specificity, with positive and negative predictive values of $90 \%$ and $22 \%$, respectively. Test accuracy was $40 \%$.

Normal values to otoacoustic emissions are already well established (SNR $<6 \mathrm{~dB}$ ), so our aim was not to identify a better cut-off point, but to find the relation between DKD and otoacoustic emissions. In this case, we have done a ROC curve and added this analysis in Fig. 3 . The best cutoff in decibels was $8.5 \mathrm{~dB}$ (above literature standard) in $8.000 \mathrm{~Hz}$ frequency band, which showed $73 \%$ of sensitivity and $40 \%$ of specificity, with $50 \%$ of accuracy.

\section{Otoacoustic emissions and autonomic neuropathy}

The results of the autonomic function tests are shown in Table 3. CAN was diagnosed in 17/37 (46\%) patients. Valsalva test was abnormal in $2 / 37(6 \%)$, deep breathing test was in 10/37 (27\%) and lying-to-standing test was normal $(73 \%)$ or borderline $(23 \%)$ in all patients. Five patients $(13 \%)$ were classified as borderline in two different tests and were included in our analyses and diagnosed with CAN.

Abnormal OAEs responses in $6 \mathrm{k} \mathrm{Hz}$ frequency band were correlated with presence $(\mathrm{r}=0.41, \mathrm{p}=0.01)$ and 
Table 2 Renal function and albuminuria in T1DM patients $(n=37)$

\begin{tabular}{lcccc}
\hline & Normoalbuminuria $(\mathbf{N}=\mathbf{1 6})$ & Microalbuminuria $(\mathbf{N}=17)$ & Macroalbuminuria $(\mathbf{N}=\mathbf{4})$ & $\mathbf{p}$ \\
\hline Serum creatinine $(\mathrm{mg} / \mathrm{dl})$ & $0.9 \pm 0.1$ & $0.9 \pm 0.2$ & $1.0 \pm 0.2$ & $\mathrm{NS}$ \\
GFR $\left(\mathrm{ml} / \mathrm{min} / 1.73 \mathrm{~m}^{2}\right)$ & $109.4 \pm 20.3$ & $94.4 \pm 23.6$ & $81.7 \pm 16.6$ & $<0.05^{\dagger}$ \\
Albuminuria $(\mathrm{mg} / 24 \mathrm{~h})$ & $23.8 \pm 8.2$ & $51.8 \pm 25.7$ & $688.6 \pm 857.0$ & $<0.05^{\ddagger}$ \\
\hline
\end{tabular}

${ }^{\dagger} \mathrm{p}<0.05$ between normoalbuminuric versus micro and macroalbumiunric patients

₹ $\mathrm{p}<0.05$ between all groups

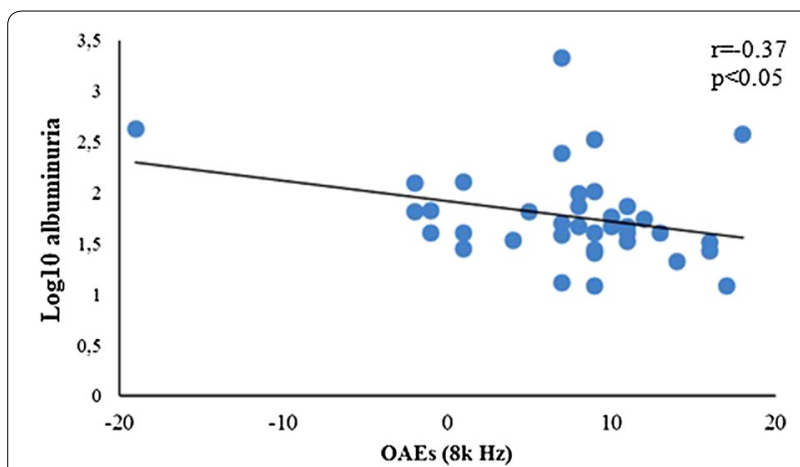

Fig. 2 Correlation between albuminuria and otoacoustic emissions (OAEs) in the frequency band of 8000 Hertz in patients with T1DM $(n=37)$

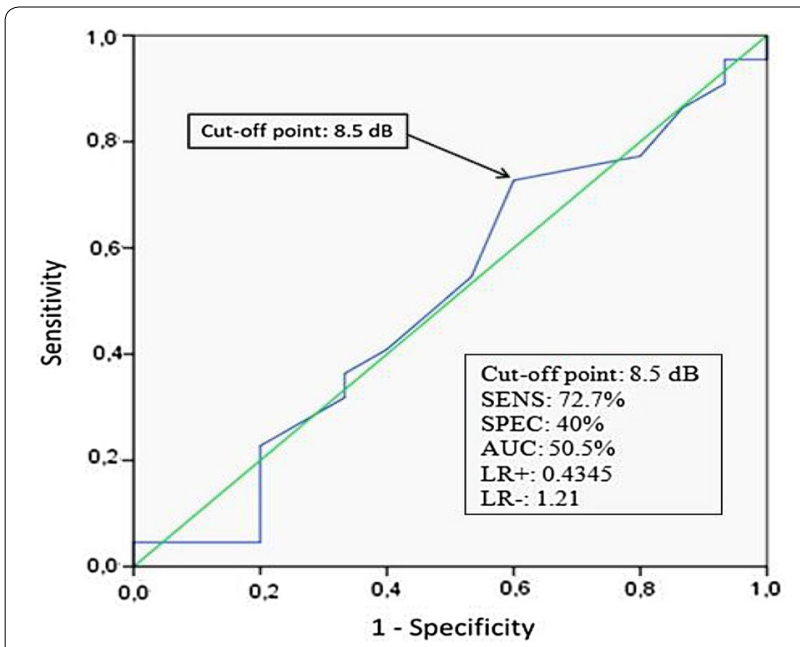

Fig. 3 ROC curve and cut-off point for the Signal Noise Ratio (SNR) and presence of DKD. Sens, sensitivity; Spec, specificity; AUC, area under the curve; $L R+$, positive likelihood ratio; LR-, negative likelihood ratio

Table 3 Results of the autonomic function tests in T1DM patients $(\mathbf{N}=\mathbf{3 7})$

\begin{tabular}{lccl}
\hline Autonomic function test & Normal & Borderline & Abnormal \\
\hline Valsalva test & $29 / 37(78 \%)$ & $6 / 37(16 \%)$ & $2 / 37(6 \%)$ \\
Deep breathing test & $24 / 37(65 \%)$ & $3 / 37(8 \%)$ & $10 / 37(27 \%)$ \\
Lying-to-standing test & $27 / 37(73 \%)$ & $10 / 37(27 \%)$ & $0 / 37$ \\
\hline
\end{tabular}

severity of CAN (CAN score) $(\mathrm{r}=0.44, \mathrm{p}<0.001)$. Additionally, $7 / 9(78 \%)$ of patients with abnormal OAEs responses in this frequency band also presented CAN.

Furthermore, otoacoustic emissions responses in $6 \mathrm{k} \mathrm{Hz}$ frequency band were correlated with results of Valsalva test $(\mathrm{r}=0.47, \mathrm{p}<0.001)$, deep breathing test $(\mathrm{r}=0.33, \mathrm{p}<0.05)$ and lying-to-standing test $(\mathrm{r}=0.32$, $\mathrm{p}=0.05)$.

Abnormal OAEs responses in low frequency bands $(0.5 \mathrm{k}$ and $1 \mathrm{k} \mathrm{Hz})$ correlated with postural BP change in lying-to-standing test $(0.5 \mathrm{k} \mathrm{Hz}: \mathrm{r}=-0.35, \mathrm{p}<0.05$; $1 \mathrm{k} \mathrm{Hz}: \mathrm{r}=-0.42, \mathrm{p}=0.01)$.

As screening test to CAN, distortion product otoacoustic emissions showed $41 \%$ of sensitivity and $90 \%$ of specificity, with positive predictive value (PPV) of $78 \%$ and negative predictive value (NPV) of $64 \%$. Test accuracy was $67 \%$.

\section{Discussion}

The present study demonstrated a high prevalence of abnormal OAE responses in T1DM patients, and also an association between this finding and presence and severity of DKD and CAN. This correlation was more evident in high frequency bands, which present greater reproducibility. In these frequencies, OAEs responses showed high specificity but low sensitivity to detection of DKD and CAN.

Several studies have demonstrated the presence of lower amplitude of OAEs in T1DM patients with normal auditory thresholds, pointing to early subclinical modifications of the inner ear caused by the metabolic changes of diabetes [28-30]. In OAEs assessment, higher frequencies $(4 \mathrm{k}, 6 \mathrm{k}$, and $8 \mathrm{k} \mathrm{Hz})$ are considered the most specific for cochlear injury diagnosis [18] and are also the most frequently affected in the diabetes-related hearing impairment $[3,6,10,31,32]$. Ottaviani et al. [33], studying 60 T1DM patients without hearing symptoms, observed abnormal OAE responses in $28 \%$ of them, in spite of a normal audiometric hearing threshold. The higher prevalence of cochlear dysfunction observed in our sample may be at least in part justified by the worse glycemic control in our patients $(\mathrm{HbA} 1 \mathrm{c}=9.4 \pm 2.6 \%$ vs $8.1 \pm 1.8 \%)$. Another explanation involves the high 
prevalence of diabetes complications in our sample, given that we are a tertiary care hospital. As abnormal OAE responses are associated with microangiopathy, it is expected to find more cochlear dysfunction in a sample with high prevalence of diffuse microvascular complications. The association between abnormal otoacoustic emissions, DKD, and CAN, described in our study, reinforces that hypothesis. Therefore, if it is true that hearing impairment is a pre-clinical stage of hearing loss [34], performing distortion product otoacoustic emissions in T1DM patients with microvascular complications could be useful to prevent auditory damage. Given that, diabetic patients should be instructed about this damage and how to prevent it, aiming to improve glycemic control [35], avoid exposure to loud noises, ear infections and tympanic membrane perforations, which can jeopardize healthy hearing of these patients [36].

Sasso et al. [37], studying patients with type 2 diabetes mellitus, have not found association between optoacoustic emissions and DKD and diabetic retinopathy. It might have occurred because of the existence of many confounders in T2DM (older age, hypertension and the use of many concomitant ototoxic medications) that could interfere in audiometric evaluation. In addition, they have included patients with history of otological pathology. In our opinion, to avoid those confounders, the relation between diabetic complications and abnormal OAE should be better studied in type 1 diabetic patients.

The relationship between diabetes and hearing dysfunction has been well established [7, 32]. In a recent meta-analysis, Horikawa et al. found a high prevalence of hearing impairment in diabetic patients [7]. Moreover, a cross-sectional study including 5140 patients, showed an occurrence of hearing impairment in 54\% of DM patients vs $32 \%$ in controls [32]. In addition, in other series, decreased distortion product otoacoustic emissions amplitudes in different frequency bands were associated with diabetic neuropathy $[5,38]$, nephropathy, and retinopathy [5]. However, these studies included people with type $2 \mathrm{DM}$, which is largely associated with other variables implicated in cochlear injuries, such as hypertension, dyslipidemia, and atherosclerosis $[10,39,40]$.

Our data shows a DKD prevalence of $88 \%$ in patients with abnormal otoacoustic responses in $8 \mathrm{k} \mathrm{Hz}$ frequency band, that was additionally associated with albuminuria. In case of a disease with several differential diagnoses, it is important to use a specific method. Analyzing our ROC curve, the best cut-off point in decibels was $8.5 \mathrm{~dB}$ (above literature standard) in $8000 \mathrm{~Hz}$ frequency band, which showed $73 \%$ of sensitivity and $40 \%$ of specificity, with $50 \%$ of accuracy. Nevertheless, to question the current otoacoustic emissions normal threshold we would need a larger group. We have also found quite similar results between CAN and abnormal OAEs responses in $6 \mathrm{k} \mathrm{Hz}$ band. Some studies, using auditory brainstem evoked and audiometric techniques, have found a high risk of hearing impairment in T1DM patients [41, 42]. Lasagni et al. [43] observed that distortion product otoacoustic emissions intensities at medium frequencies $(2.8-4 \mathrm{kHz})$ were significantly lower in T1DM, however, the study did not show an association between abnormal OAE responses and microvascular alterations, such as albuminuria and retinopathy. Nevertheless, Lisowska et al. were the only ones that assessed this issue in T1DM patients, using distortion product otoacoustic emissions in high frequencies. They performed a cohort study with 42 T1DM patients and have not found an association between DKD, retinopathy and abnormal otoacoustic emissions responses in high frequency bands [4]. This may have occurred because they have not searched OAEs at $8 \mathrm{k} \mathrm{Hz}$ frequency and no CAN screening was done. As we are aware, this is the first study to suggest an association between abnormal otoacoustic responses, especially in high frequency bands, and presence and severity of DKD and CAN in T1DM patients without auditory symptoms. However, our data should be interpreted carefully. Due to overlapping clinical features of glomerulopathies, DKD is often diagnosed in diabetic patients without further research. In 2013, Sharma et al. performed renal biopsy in 611 diabetic patients and found that only 227 (37\%) had DKD alone, while 220 (36\%) had nondiabetic renal disease solely, and 164 (27\%) had both [22].

Retinopathy constitutes an independent risk marker for DKD, given its high sensitivity. Therefore, in diabetic patients with albuminuria but without retinopathy, renal biopsy is indicated to establish whether this laboratory finding can be attributed to diabetes [44]. In this scenario, performing distortion product otoacoustic emissions could help. Due to its high specificity, if it is abnormal in $8 \mathrm{k} \mathrm{Hz}$ band, the probability of DKD diagnosis is very high, so this procedure could be avoided. In fact, it is well established that loss of hair cells tend to occur in patients $>15$ years of diabetes duration [45]; while DKD is typically developed after 10 years of T1DM diagnosis [27]. In other words, DM affects kidneys before cochlea, it could be the explanation why distortion product otoacoustic emissions is a highly specific but not too sensible test for DKD diagnosis. Larger studies are necessary to confirm this data.

Furthermore, in our study, $78 \%$ of the patients with a lower amplitude of OAEs in the $6 \mathrm{k} \mathrm{Hz}$ frequency band had abnormal autonomic function tests, suggesting that the otoacoustic emissions study in high frequencies also presents high specificity for the diagnosis of CAN. Friedman et al. [46] suggested that microangiopathy could be nonspecific rather than a causal finding, and Makashima and 
Tanaka [47] described atrophy of spiral ganglion neurons and demyelination of the auditory nerve in a few diabetic subjects. Demyelination is also the initial lesion in peripheral nerves of diabetic extremities, which could imply that hearing impairment can possibly be due to diabetic autonomic neuropathy of the auditory nerve. Di Leo et al. show in a study with $48 \mathrm{DM}$ patients that OAE is reduced in patients with neuropathy compared to those without [48]. Therefore, one possible explanation is that autonomic neuropathy could be a via through which microvascular disease could lead to hearing impairment in diabetic patients.

Finally, the main limitation of our study was the small number of patients evaluated, although the analyzed frequencies are highly reproducible. Other weaknesses are the variables besides DM that could implicate in cochlear injuries, such as age, hypertension, dyslipidemia, smoking, excessive exposure to noise and use of ototoxic drugs. However, in our study, these confounders were minimum, since the presence of otological pathologies was an exclusion criterion, the population was young (average age of 23.5 years old), and there was a low prevalence of hypertension, dyslipidemia, and smoking.

\section{Conclusion}

Our results suggest that abnormal otoacoustic emissions responses in high frequency bands are associated with diabetes microvascular complications and could be a risk marker for DKD and CAN, presenting low sensitivity and high specificity. However, this data should be confirmed in a larger sample. Therefore, assuming that hearing impairment is a pre-clinical stage of hearing loss [35], performing distortion product otoacoustic emissions in T1DM patients with microvascular complications could be useful to identify those who would be benefited with regular audiologic follow up and tighter glycemic control.

\section{Article summary \\ Strengths and limitations of this study Strengths}

The great reproducibility of OAE responses in high frequency bands that showed high specificity to detection of DKD and CAN. In addition, confounders were minimum, since the presence of otological pathologies was an exclusion criterion, the population was young (average age of 23.5 years old), and there was a low prevalence of hypertension, dyslipidemia, and smoking

\section{Limitations}

The small number of patients evaluated and the possible presence of variables besides DM that could implicate in cochlear injuries, such as age, hypertension, dyslipidemia and smoking

\section{Abbreviations}

T1DM: type 1 diabetes mellitus; OAE: otoacoustic emissions; DPOAE: distortion product otoacoustic emissions; DKD: diabetes kidney disease; CAN: cardiac autonomic neuropathy; ADA: American Diabetes Association; DM: diabetes mellitus; BMI: body max index; HPLC: high performance liquid chromatography; MDRD: modification of diet in renal disease; FPG: fasting plasma glucose; TSH: thyroid-stimulating hormone; FT4: free thyroxine.

\section{Authors' contributions}

All persons who meet authorship criteria are listed as authors, and all authors certify that they have participated sufficiently in the work to take public responsibility for the content, including participation in the concept, design, analysis, writing, or revision of the manuscript. CCK and JSF took part in conception and design of study. KMF, LSDS, NNMQ, ALA, MCS, LCJ, CLLPM, ACCBS and FTCM were responsible for acquisition of data, while NAZ, WMS, FMS, FSR, LDA and JFAN have done the analysis and interpretation of data. MNL, LMC, NALM, NJKSN, IIFF and MCNIO have drafted the manuscript together. All authors read and approved the final manuscript.

\section{Acknowledgements}

The authors thank Pró-Reitoria de Pesquisa (PROPESP) of Federal University of Pará and Programa de Pós-Graduação em Oncologia e Ciências Médicas of University Hospital João de Barros Barreto-Endocrinology Division, Federal University of Pará, for the assistance.

\section{Competing interests}

The authors declare that they have no competing interests.

\section{Availability of data and materials}

The datasets during and/or analyzed during the current study available from the corresponding author on reasonable request.

\section{Funding statement}

The authors received no specific funding for this work.

\section{Statement of informed consent}

Informed consent was obtained from all patients for being included in the study.

\section{Statement of human and animal rights}

All procedures followed were in accordance with the ethical standards of the responsible committee on human experimentation (institutional and national) and with the Helsinki Declaration of 1975, as revised in 2008. This study was approved by University Hospital Joao de Barros Barreto ethics committee.

\section{Publisher's Note}

Springer Nature remains neutral with regard to jurisdictional claims in published maps and institutional affiliations.

Received: 30 August 2018 Accepted: 19 October 2018

Published online: 09 November 2018

\section{References}

1. Mokshagundam SPL, Broadstone VL. Diabetes Mellitus. Primary care in obstetrics and gynecology. New York: Springer; 2007. p. 309-25. https:// doi.org/10.1007/978-0-387-32328-2_15.

2. Eren E, Harman E, Arslanoğlu S, Önal K. Effects of type 2 diabetes on otoacoustic emissions and the medial olivocochlear reflex. Otolaryngol Neck Surg. 2014;150(6):1033-9. https://doi.org/10.1177/0194599814527574.

3. de León-Morales LVD, Jáuregui-Renaud K, Garay-Sevilla ME, HernándezPrado J, Malacara-Hernández JM. Auditory impairment in patients with type 2 diabetes mellitus. Arch Med Res. 2005;36(5):507-10. https://doi. org/10.1016/j.arcmed.2005.02.002.

4. Lisowska G, Namysłowski G, Morawski K, Strojek K. Cochlear dysfunction and diabetic microangiopathy. Scand Audiol. 2001;30(1):199-203. https:// doi.org/10.1080/010503901300007524. 
5. Sugimoto S, Teranishi M, Fukunaga Y, Yoshida T, Sugiura S, Uchida Y, et al. Contributing factors to hearing of diabetic patients in an in-hospital education program. Acta Otolaryngol. 2013;133(11):1165-72. https://doi. org/10.3109/00016489.2013.803599.

6. Calvin D, Watley SR. Diabetes and hearing loss among underserved populations. Nurs Clin North Am. 2015;50(3):449-56. https://doi.org/10.1016/j. cnur.2015.05.001.

7. Horikawa C, Kodama S, Tanaka S, Fujihara K, Hirasawa R, Yachi Y, et al. Diabetes and risk of hearing impairment in adults: a meta-analysis. J Clin Endocrinol Metab. 2013;98(1):51-8. https://doi.org/10.1210/jc.2012-2119.

8. Kurien M, Thomas K, Bhanu TS. Hearing threshold in patients with diabetes mellitus. J Laryngol Otol. 1989;103(02):164-8. https://doi.org/10.1017/ s0022215100108345.

9. Konrad-Martin D, Reavis KM, Austin D, Reed N, Gordon J, McDermott D, et al. Hearing impairment in relation to severity of diabetes in a veteran cohort. Ear Hear. 2015;36(4):381-94. https://doi.org/10.1097/aud.00000 00000000137.

10. Kakarlapudi V, Sawyer R, Staecker H. The effect of diabetes on sensorineural hearing loss. Otol Neurotol. 2003;24(3):382-6. https://doi. org/10.1097/00129492-200305000-00006.

11. Hong O, Buss J, Thomas E. Type 2 diabetes and hearing loss. Dis Mon. 2013;59(4):139-46. https://doi.org/10.1016/.disamonth.2013.01.004

12. Kurt E, Öztürk F, Günen A, Sadikoglu Y, Sari RA, Yoldas TK, et al. Relationship of retinopathy and hearing loss in type 2 diabetes mellitus. Ann Ophthalmol. 2002;34(3):216-22. https://doi.org/10.1007/s1200 9-002-0026-4.

13. Lisowska G, Namyslowski G, Morawski K, Strojek K. Otoacoustic emissions and auditory brain stem responses in insulin dependent diabetic patients. Otolaryngol Pol. 2002;56(2):217-25.

14. Kemp DT, Ryan S, Bray P. A guide to the effective use of otoacoustic emissions. Ear Hear. 1990;11(2):93-105. https://doi.org/10.1097/00003 446-199004000-00004.

15. Lonsbury-Martin BL, Martin GK. The clinical utility of distortion-product otoacoustic emissions. Ear Hear. 1990;11(2):144-54. https://doi. org/10.1097/00003446-199004000-00009

16. Fernandes LC, Casais-Silva L, Ladeia AM. Dysfunction of the peripheral and central auditory pathway in patients with type 1 diabetes mellitus. J Diabetes Mellitus. 2012;02(01):76-81. https://doi.org/10.4236/ jdm.2012.21012.

17. Hao J, Fu X, Zhang C, Zhang X, Zhao S, Li Y. Early detection of hearing impairment in patients with diabetes mellitus with otoacoustic emission. A systematic review and meta-analysis. Acta Otolaryngol. 2016;137(2):179-85. https://doi.org/10.1080/00016489.2016.1223344.

18. Kemp DT. Otoacoustic emissions, their origin in cochlear function, and use. Br Med Bull. 2002;63(1):223-41. https://doi.org/10.1093/ bmb/63.1.223.

19. Hall JW. Handbook of otoacoustic emissions. Ear Hear. 2000;21 (6):646. https://doi.org/10.1097/00003446-200012000-00013.

20. Gorga MP, Neely ST, Ohlrich B, Hoover B, Redner J, Peters J. From laboratory to clinic: a large scale study of distortion product otoacoustic emissions in ears with normal hearing and ears with hearing loss. Ear Hear. 1997;18(6):440-55. https://doi.org/10.1097/00003446-199712000-00003.

21. Tuttle KR, Bakris GL, Bilous RW, Chiang JL, de Boer IH, Goldstein-Fuchs J, et al. Diabetic kidney disease: a report from an ADA consensus conference. Diabetes Care. 2014;37(10):2864-83. https://doi.org/10.2337/ dc14-1296.

22. Sharma SG, Bomback AS, Radhakrishnan J, Herlitz LC, Stokes MB, Markowitz GS, et al. The modern spectrum of renal biopsy findings in patients with diabetes. Clin J Am Soc Nephrol. 2013;8(10):1718-24.

23. Ewing DJ, Boland O, Neilson JMM, Cho CG, Clarke BF. Autonomic neuropathy, QT interval lengthening, and unexpected deaths in male diabetic patients. Diabetologia. 1991;34(3):182-5. https://doi.org/10.1007/bf004 18273.

24. Valensi P, Sachs R-N, Harfouche B, Lormeau B, Paries J, Cosson E, et al. Predictive value of cardiac autonomic neuropathy in diabetic patients with or without silent myocardial ischemia. Diabetes Care. 2001;24(2):339-43. https://doi.org/10.2337/diacare.24.2.339.

25. Nussinovitch U, Katz U, Nussinovitch M, Nussinovitch N. Deep breath test for evaluation of autonomic nervous system dysfunction in familial dysautonomia. Isr Med Assoc J. 2009;1 1(10):615-8.
26. Valensi PE, Johnson NB Maison-Blanche P Extramania F Motte G, Coumel $P$. Influence of cardiac autonomic neuropathy on heart rate dependence of ventricular repolarization in diabetic patients. Diabetes Care. 2002;25(5):918-23. https://doi.org/10.2337/diacare.25.5.918.

27. Professional Practice Committee: Standards of Medical Care in Diabetes-2018. Vol. 41, Diabetes care. United States; 2018. p. S3.

28. Teng Z, Tian $\mathrm{R}$, Xing $\mathrm{F}$, Tang $\mathrm{H}$, Xu J, Zhang $\mathrm{B}$, et al. An association of type 1 diabetes mellitus with auditory dysfunction: a systematic review and meta-analysis. Laryngoscope. 2017;127(7):1689-97. https://doi. org/10.1002/lary.26346.

29. Hilali A, Das V, Boulton A. A study of otoacoustic emissions in type 1 diabetes mellitus. Audiol Med. 2003;1 (4):255-60. https://doi. org/10.1080/16513860310022372

30. ALDajani N, ALkurdi A, ALMutair A, ALdraiwesh A, ALMazrou KA. Is type 1 diabetes mellitus a cause for subtle hearing loss in pediatric patients? Eur Arch Otorhinolaryngol. 2015;272(8):1867-71.

31. Vaughan N, James K, McDermott D, Griest S, Fausti S. A 5-year prospective study of diabetes and hearing loss in a veteran population. Otol Neurotol. 2006;27(1):37-43. https://doi.org/10.1097/01.mao.0000194812.69556.74.

32. Bainbridge KE, Hoffman HJ, Cowie CC. Diabetes and hearing impairment in the united states: audiometric evidence from the national health and nutrition examination survey, 1999 to 2004. Ann Intern Med. 2008;149(1):1. https://doi.org/10.7326/0003-4819-149-1-20080701000231.

33. Ottaviani F, Dozio N, Neglia CB, Riccio S, Scavini M. Absence of otoacoustic emissions in insulin-dependent diabetic patients. J Diabetes Complications. 2002;16(5):338-43. https://doi.org/10.1016/s1056-8727(01)00224-0.

34. Botelho CT, Carvalho SA, Silva IN. Increased prevalence of early cochlear damage in young patients with type 1 diabetes detected by distortion product otoacoustic emissions. Int J Audiol. 2014;53(6):402-8.

35. Yang $\mathrm{C}-\mathrm{H}$, Schrepfer T, Schacht J. Age-related hearing impairment and the triad of acquired hearing loss. Front Cell Neurosci. 2015. https://doi. org/10.3389/fncel.2015.00276

36. Hong O, Kerr MJ, Poling GL, Dhar S. Understanding and preventing noiseinduced hearing loss. Dis Mon. 2013;59(4):110-8.

37. Sasso FC, Salvatore T, Tranchino G, Cozzolino D, Caruso AA, Persico M, et al. Cochlear dysfunction in type 2 diabetes: a complication independent of neuropathy and acute hyperglycemia. Metabolism. 1999:48:134650. https://doi.org/10.1016/s0026-0495(99)90141-5.

38. Lisowska G, Namysowski G, Morawski K, Strojek K. Early identification of hearing impairment in patients with type 1 diabetes mellitus. Otol Neurotol. 2001:22(3):316-20. https://doi.org/10.1097/00129492-20010 5000-00008.

39. Esparza CM, Jáuregui-Renaud K, Morelos CMC, Muhl GEA, Mendez MN, Carillo NS, et al. Systemic high blood pressure and inner ear dysfunction: a preliminary study. Clin Otolaryngol. 2007;32(3):173-8. https://doi.org/10 $.1111 / j .1365-2273.2007 .01442 . x$

40. Erdem T, Ozturan O, Miman MC, Ozturk C, Karatas E. Exploration of the early auditory effects of hyperlipoproteinemia and diabetes mellitus using otoacoustic emissions. Eur Arch Otorhinolaryngol. 2003:260(2):62-6.

41. Varkonyi TT, Toth F, Rovo L, Lengyel C, Kiss JG, Kempler P, et al. Impairment of the auditory brainstem function in diabetic neuropathy. Diabetes Care. 2002;25(3):631-2. https://doi.org/10.2337/diacare.25.3.631.

42. Goldsher M, Pratt H, Hassan A, Shenhav R, Eliachar I, Kanter Y. Auditory brainstem evoked potentials in insulin-dependent diabetics with and without peripheral neuropathy. Acta Otolaryngol. 1986;102(3-4):204-8. https://doi.org/10.3109/00016488609108667.

43. Lasagni A, Giordano P, Lacilla M, Raviolo A, Trento M, Camussi E, et al. Cochlear, auditory brainstem responses in Type 1 diabetes: relationship with metabolic variables and diabetic complications. Diabet Med. 2015:33(9):1260-7. https://doi.org/10.1111/dme.13039.

44. Kidney Disease: Improving Global Outcomes (KDIGO) CKD Work Group. KDIGO 2012 clinical practice guideline for the evaluation and management of chronic kidney disease. Kidney Inter Suppl. 2013;3:1-150.

45. Fukushima H, Cureoglu S, Schachern PA, Kusunoki T, Oktay MF, Fukushima $\mathrm{N}$, et al. Cochlear changes in patients with type 1 diabetes mellitus. Otolaryngol Neck Surg. 2005;133(1):100-6. https://doi.org/10.1016/j.otohn s.2005.02.004. 
46. Friedman SA. Hearing and diabetic neuropathy. Arch Intern Med. 1975;135(4):573. https://doi.org/10.1001/archinte.1975.00330040085014.

47. Makishima K, Tanaka K. Pathological changes of the inner ear and central auditory pathway in diabetics. Ann Otol Rhinol Laryngol. 1971;80(2):21828. https://doi.org/10.1177/000348947108000208.
48. Di Leo MA, Di Nardo W, Cercone S, Ciervo A, Lo Monaco M, Greco AV, et al. Cochlear dysfunction in IDDM patients with subclinical peripheral neuropathy. Diabetes Care. 1997;20(5):824-8.
Ready to submit your research? Choose BMC and benefit from:

- fast, convenient online submission

- thorough peer review by experienced researchers in your field

- rapid publication on acceptance

- support for research data, including large and complex data types

- gold Open Access which fosters wider collaboration and increased citations

- maximum visibility for your research: over 100M website views per year

At BMC, research is always in progress.

Learn more biomedcentral.com/submissions 